\title{
Correction to: Managing pollution from antibiotics manufacturing: charting actors, incentives and disincentives
}

\author{
Niels Nijsingh ${ }^{1,2,3}$, Christian Munthe $e^{1,2}$ and D. G. Joakim Larsson ${ }^{1,4^{*}}$
}

Correction to: Environ Health (2019) 18:95 https://doi.org/10.1186/s12940-019-0531-1

Following publication of the original article [1], the author explained that there are multiple errors in the original article;

1. Incorrect Additional file 1 was uploaded and is replaced with the correct Additional file 1.

2. Several references (52-70) were incorrectly placed in the references list and they are now have been removed and placed in the supplemental material.

3. The format of Table 1 was incorrect formatted and this is now corrected.

Additional File 1 and Table 1 are included in the correction article. The original article has been corrected.

\section{Supplementary information}

Supplementary information accompanies this paper at https://doi.org/10. 1186/s12940-019-0545-8.

Additional file 1. Relevant actor types and their interests.
Published online: 12 December 2019

\section{Reference}

1. Nijsingh $\mathrm{N}$, et al. Managing pollution from antibiotics manufacturing: charting actors, incentives and disincentives. Environ Health. 2019;18:95. https://doi.org/10.1186/s12940-019-0531-1.

\footnotetext{
Author details

'Centre for Antibiotic Resistance Research (CARe), at University of Gothenburg, Gothenburg, Sweden. ${ }^{2}$ Department of Philosophy, Linguistics and Theory of Science, University of Gothenburg, Gothenburg, Sweden. ${ }^{3}$ Institute of Ethics, History and Theory of Medicine, Ludwig Maximilian University, Munich, Germany. ${ }^{4}$ Department of Infectious Diseases, Institute of Biomedicine, The Sahlgrenska Academy, University of Gothenburg, Gothenburg, Sweden.

The original article can be found online at https://doi.org/10.1186/s12940019-0531-1

* Correspondence: joakim.larsson@fysiologi.gu.se

${ }^{1}$ Centre for Antibiotic Resistance Research (CARe), at University of Gothenburg, Gothenburg, Sweden

${ }^{4}$ Department of Infectious Diseases, Institute of Biomedicine, The Sahlgrenska Academy, University of Gothenburg, Gothenburg, Sweden

Full list of author information is available at the end of the article
}

(c) The Author(s). 2019 Open Access This article is distributed under the terms of the Creative Commons Attribution 4.0 International License (http://creativecommons.org/licenses/by/4.0/), which permits unrestricted use, distribution, and reproduction in any medium, provided you give appropriate credit to the original author(s) and the source, provide a link to the Creative Commons license, and indicate if changes were made. The Creative Commons Public Domain Dedication waiver (http://creativecommons.org/publicdomain/zero/1.0/) applies to the data made available in this article, unless otherwise stated. 
Table 1 Thirty-three actor types with possibilities to contribute to the reduction of antibiotic emissions from manufacturing. For each actor type, examples of their interests, possible actions and incentives and disincentives for those actions are listed (more details to be found in the main text and the supplementary material). Broadly common interests among actors, e.g. individual's/ employees desire to contribute to positive societal change (reducing pollution, improving public health) are implicit but have not been listed for each actor. For actors \#15,16,17, 21 and 22 we have listed Swedish examples of actors types

\begin{tabular}{|c|c|c|c|c|}
\hline Actors & Interests & Actions & Incentives & Disincentives \\
\hline $\begin{array}{l}\text { \# } 1 \text { Research-based pharma- } \\
\text { ceutical companies }\end{array}$ & $\begin{array}{l}\text { - Increase } \\
\text { turnover, reduce } \\
\text { costs; } \\
\text { - Strategic } \\
\text { interests (e.g. } \\
\text { "stay ahead of } \\
\text { the curve); } \\
\text { - Reputation } \\
\text { concerns; } \\
\text { - Preserve } \\
\text { effectiveness of } \\
\text { product by } \\
\text { curbing } \\
\text { antibiotic } \\
\text { resistance. }\end{array}$ & $\begin{array}{l}\text {-Motivate \#3: demand good } \\
\text { pollution control for the API's } \\
\text { they buy; } \\
\text { - Monitor \& reduce their own } \\
\text { discharges; } \\
\text { - Set internal discharge limits; } \\
\text { - Act transparently with } \\
\text { regards to production sites } \\
\text { (also of suppliers) and } \\
\text { environmental performance. }\end{array}$ & $\begin{array}{l}\text { - Emission standards; } \\
\text { - Legal requirements; } \\
\text { - Economic incentives (price, } \\
\text { costs, turnover); } \\
\text { - Pressure from investors } \\
\text { and buyers; } \\
\text { - Reputation concerns. }\end{array}$ & $\begin{array}{l}\text { - Transparency as a threat for } \\
\text { reputation concerns; } \\
\text { - Higher production cost; } \\
\text { - Lack of follow-up of external } \\
\text { demands - risks of unfair } \\
\text { competition. }\end{array}$ \\
\hline $\begin{array}{l}\text { \#2 Generic pharmaceutical } \\
\text { companies }\end{array}$ & $\begin{array}{l}\text { - Increase } \\
\text { turnover, reduce } \\
\text { costs; } \\
\text { - Strategic } \\
\text { interests (e.g. } \\
\text { "stay ahead of } \\
\text { the curve"); } \\
\text { - Preserve } \\
\text { effectiveness of } \\
\text { product by } \\
\text { curbing } \\
\text { antibiotic } \\
\text { resistance. }\end{array}$ & $\begin{array}{l}\text {-Motivate \#3: demand good } \\
\text { pollution control for the API's } \\
\text { they buy; } \\
\text { - Monitor \& reduce their own } \\
\text { discharges; } \\
\text { - Set internal discharge limits; } \\
\text { - Act transparently with } \\
\text { regards to production sites } \\
\text { (also of suppliers) and } \\
\text { environmental performance. }\end{array}$ & $\begin{array}{l}\text { - Emission standards; } \\
\text { - Legal requirements; } \\
\text { - Economic incentives (price, } \\
\text { costs, turnover); } \\
\text { - Pressure from investors } \\
\text { and buyers. }\end{array}$ & $\begin{array}{l}\text { - Higher production cost; } \\
\text { - Lack of follow-up of external } \\
\text { demands - risks of unfair } \\
\text { competition; }\end{array}$ \\
\hline $\begin{array}{l}\text { \# } 3 \text { Subcontracting } \\
\text { pharmaceutical companies }\end{array}$ & $\begin{array}{l}\text { - Increase } \\
\text { turnover, reduce } \\
\text { costs; } \\
\text { - Strategic } \\
\text { interests (e.g. } \\
\text { "stay ahead of } \\
\text { the curve"); } \\
\text { - Preserve } \\
\text { effectiveness of } \\
\text { product by } \\
\text { curbing } \\
\text { antibiotic } \\
\text { resistance. }\end{array}$ & $\begin{array}{l}\text { - Monitor and reduce their } \\
\text { own discharges; } \\
\text { - Set internal discharge limits; } \\
\text { - Act transparently with } \\
\text { regards to environmental } \\
\text { performance. }\end{array}$ & $\begin{array}{l}\text { - Emission standards; } \\
\text { - Legal requirements; } \\
\text { - Economic incentives (price, } \\
\text { costs, turnover); } \\
\text { - Pressure from investors } \\
\text { and buyers (i.e. \#1 \& \#2). }\end{array}$ & $\begin{array}{l}\text { - Higher production cost; } \\
\text { - Lack of follow-up of external } \\
\text { demands - risks of unfair } \\
\text { competition; }\end{array}$ \\
\hline $\begin{array}{l}\text { \# } 4 \text { Umbrella organisations/ } \\
\text { collaborations between } \\
\text { pharmaceutical companies. }\end{array}$ & $\begin{array}{l}\text { - Represent } \\
\text { members (\#1, } \\
\# 2, \# 3 \text { ); } \\
\text { - Align interests of } \\
\text { members. }\end{array}$ & -Coordinate action & $\begin{array}{l}\text { - In addition to those } \\
\text { applying to } \# 1,2 \text { and } 3 \text { : } \\
\text { become a stronger force } \\
\text { for promoting common } \\
\text { interests. }\end{array}$ & $\begin{array}{l}\text { - Interest and priorities may } \\
\text { differ between members. }\end{array}$ \\
\hline $\begin{array}{l}\text { \#5 Owners of pharmaceutical } \\
\text { companies }\end{array}$ & $\begin{array}{l}\text { - Profit on } \\
\text { investment; } \\
\text { - Reputation } \\
\text { concerns. }\end{array}$ & $\begin{array}{l}\text { - (Threaten to) withdraw } \\
\text { investments in } \# 1, \# 2 \text { and } \# 3 \text {; } \\
\text { - Power through } \\
\text { representation in boards. }\end{array}$ & $\begin{array}{l}\text { - Pressure from customers } \\
\text { and interest groups; } \\
\text { - Financial incentives (risk } \\
\text { for loss of business } \\
\text { associated with "scandals"). }\end{array}$ & $\begin{array}{l}\text { - Limits on (short-term) profits, } \\
\text { as owners set profit } \\
\text { expectations. }\end{array}$ \\
\hline $\begin{array}{l}\text { \# } 6 \text { Waste water treatment } \\
\text { plants (WWTPs) }\end{array}$ & $\begin{array}{l}\text { - Increase } \\
\text { turnover, reduce } \\
\text { costs. }\end{array}$ & $\begin{array}{l}\text { - Implement more effective } \\
\text { treatment; } \\
\text { - Monitor and report } \\
\text { emissions. }\end{array}$ & $\begin{array}{l}\text { - Government legislation; } \\
\text { - Subsidies. }\end{array}$ & - Costs. \\
\hline \# 7 Parallel importers & $\begin{array}{l}\text { - Increase } \\
\text { turnover, reduce } \\
\text { costs. }\end{array}$ & $\begin{array}{l}\text { - Promote transparency and } \\
\text { regulations. }\end{array}$ & $\begin{array}{l}\text { - Pressure from buyers; } \\
\text { - Legislation. }\end{array}$ & $\begin{array}{l}\text { - Very limited ability to gain } \\
\text { information on, or to influence } \\
\text { the production chain }\end{array}$ \\
\hline \# 8 Producing country states & - Represent & -Regulate industry in terms of & - Political pressure from & - Economic interests: protecting \\
\hline
\end{tabular}


Table 1 Thirty-three actor types with possibilities to contribute to the reduction of antibiotic emissions from manufacturing. For each actor type, examples of their interests, possible actions and incentives and disincentives for those actions are listed (more details to be found in the main text and the supplementary material). Broadly common interests among actors, e.g. individual's/ employees desire to contribute to positive societal change (reducing pollution, improving public health) are implicit but have not been listed for each actor. For actors \#15,16,17, 21 and 22 we have listed Swedish examples of actors types (Continued)

\begin{tabular}{|c|c|c|c|c|}
\hline Actors & Interests & Actions & Incentives & Disincentives \\
\hline & $\begin{array}{l}\text { population; } \\
\text { - Protect public } \\
\text { health; } \\
\text { - Protect } \\
\text { economic } \\
\text { interests. }\end{array}$ & $\begin{array}{l}\text { emissions; } \\
\text {-Pressure, negotiate with \#1-3; } \\
\text { - Sponsor research and } \\
\text { knowledge transfer; } \\
\text { - Support infrastructure. }\end{array}$ & $\begin{array}{l}\text { citizens and interest } \\
\text { groups; } \\
\text { - Treaties, multilateral } \\
\text { agreements, foreign } \\
\text { pressure; } \\
\text { - Public health concerns. }\end{array}$ & $\begin{array}{l}\text { current industry - strict } \\
\text { standards may create } \\
\text { disadvantages for national } \\
\text { producers; } \\
\text { - Lobbying by \#1-3. }\end{array}$ \\
\hline $\begin{array}{l}\text { \# } 9 \text { Environmental oversight } \\
\text { agencies }\end{array}$ & $\begin{array}{l}\text { - Follow statutes } \\
\text { and directives as } \\
\text { defined by \#8; } \\
\text { - Protect the } \\
\text { environment } \\
\text { (and public } \\
\text { health) }\end{array}$ & $\begin{array}{l}\text { - Implement and enforce rules } \\
\text { and regulations; } \\
\text {-Provide data on emissions. }\end{array}$ & $\begin{array}{l}\text { - Pressure from various } \\
\text { actors; } \\
\text { - Directives deriving from } \\
\text { \#8. }\end{array}$ & $\begin{array}{l}\text { - Pressure from \#1-3, in } \\
\text { particular on the local level. }\end{array}$ \\
\hline \# 10 Citizens of producer states & $\begin{array}{l}\text { - Economic } \\
\text { concerns; } \\
\text { - Public health;- } \\
\text { environmental } \\
\text { protection. }\end{array}$ & $\begin{array}{l}\text {-Pressure industry and } \\
\text { government; } \\
\text {-Vote. }\end{array}$ & $\begin{array}{l}\text { - Awareness; } \\
\text { - Economic, health and } \\
\text { environmental interests. }\end{array}$ & $\begin{array}{l}\text { - Lack of information/ awareness; } \\
\text { - Lack of interest; } \\
\text { - Lack of effective political } \\
\text { power. }\end{array}$ \\
\hline $\begin{array}{l}\text { \# } 11 \text { Citizen interest groups, } \\
\text { environmental and human } \\
\text { rights NGOs. }\end{array}$ & $\begin{array}{l}\text { - Represent \#10; } \\
\text { - Represent } \\
\text { particular } \\
\text { interests. }\end{array}$ & $\begin{array}{l}\text { - Coordinate action; } \\
\text { - Create awareness; } \\
\text { - Exert pressure. }\end{array}$ & $\begin{array}{l}\text { - Pressure from supporters; } \\
\text { - 'Mediagenic' action may } \\
\text { be more attractive with an } \\
\text { eye on public support. }\end{array}$ & - Limited political power. \\
\hline $\begin{array}{l}\text { \# } 12 \text { Inter-governmental polit- } \\
\text { ical forums (eg. G7) }\end{array}$ & $\begin{array}{l}\text { - Coordinate and } \\
\text { represent } \\
\text { national and } \\
\text { international } \\
\text { interests. }\end{array}$ & $\begin{array}{l}\text { - Apply political pressure; } \\
\text { - Harmonize policies. }\end{array}$ & $\begin{array}{l}\text { - Input by goverments, } \\
\text { political leaders; } \\
\text { - Pressure by interest } \\
\text { groups, political } \\
\text { organisations etc. }\end{array}$ & $\begin{array}{l}\text { - Many different interests, they } \\
\text { may not always align. }\end{array}$ \\
\hline \# 13 United Nations agencies & $\begin{array}{l}\text { - Initiate and } \\
\text { harmonize } \\
\text { collective action } \\
\text { on global } \\
\text { problems. }\end{array}$ & $\begin{array}{l}\text { - Create awareness; } \\
\text { - Harmonize policies across } \\
\text { nations; } \\
\text { - Exert pressure on industry } \\
\text { and governments. }\end{array}$ & $\begin{array}{l}\text { - Pressure from } \\
\text { governments, interest } \\
\text { groups, political } \\
\text { organisations etc. }\end{array}$ & - Limited power. \\
\hline \#14 Consumer country states & $\begin{array}{l}\text { - Represent } \\
\text { population; } \\
\text { - Protect public } \\
\text { health; } \\
\text { - Protect } \\
\text { economic } \\
\text { interests. }\end{array}$ & $\begin{array}{l}\text { - Regulate; } \\
\text { - Establish premiums; } \\
\text { - Direct research funding; } \\
\text { - Direct actions by national } \\
\text { agencies; } \\
\text {-Influence other consumer } \\
\text { states and \# } 30 .\end{array}$ & $\begin{array}{l}\text { - Political pressure by } \\
\text { citizens and interest } \\
\text { groups; } \\
\text { - Treaties, multilateral } \\
\text { agreements, foreign } \\
\text { pressure; } \\
\text { - (Global) public health. } \\
\text { Concerns. }\end{array}$ & $\begin{array}{l}\text { - Economic interests: costs } \\
\text { - Lobbying by \#1-3; } \\
\text { - Little mass, individually (higher } \\
\text { cost to establishing premiums); } \\
\text { - Institutional barriers (eg. state } \\
\text { generic substitution system). }\end{array}$ \\
\hline $\begin{array}{l}\text { \# } 15 \text { National Licensing } \\
\text { agencies (Läkemedelsverket, LV) }\end{array}$ & $\begin{array}{l}\text { - Follow statutes } \\
\text { and directives as } \\
\text { defined by } \# 14 ; \\
\text { - Good, affordable } \\
\text { health care. }\end{array}$ & $\begin{array}{l}\text { - Implement standards and } \\
\text { licensing of medical } \\
\text { products; }\end{array}$ & $\begin{array}{l}\text { - Steering by national } \\
\text { government. }\end{array}$ & - Limited mandate. \\
\hline $\begin{array}{l}\text { \# } 16 \text { Agencies committed to } \\
\text { subsidizing decisions (Tand- } \\
\text { och läkemedelsfömånsverket, } \\
\text { TLV) }\end{array}$ & $\begin{array}{l}\text { - Follow statutes } \\
\text { and directives as } \\
\text { defined by \#14; } \\
\text { - Good, affordable } \\
\text { health care. } \\
\text { - Effective } \\
\text { resource } \\
\text { allocation. }\end{array}$ & $\begin{array}{l}\text { Potentially (but not currently): } \\
\text { - Weigh environmental } \\
\text { concerns in reimbursement } \\
\text { decisions. }\end{array}$ & $\begin{array}{l}\text { - Steering by national } \\
\text { government. }\end{array}$ & $\begin{array}{l}\text { - Limited mandate; } \\
\text { - Limited possibilities for action } \\
\text { under current statutes. }\end{array}$ \\
\hline $\begin{array}{l}\text { \# } 17 \text { Agencies committed to } \\
\text { prescription policies } \\
\text { (Socialstyrelsen, SoS, and } \\
\text { Inspektionen för vård och } \\
\text { omsorg, IVO }\end{array}$ & $\begin{array}{l}\text { - Follow statutes } \\
\text { and directives as } \\
\text { defined by \#14; } \\
\text { - Good, affordable } \\
\text { health care. }\end{array}$ & $\begin{array}{l}\text { - Issue national treatment } \\
\text { guidelines (in cooperation } \\
\text { with \# 18). }\end{array}$ & $\begin{array}{l}\text { - Steering by national } \\
\text { government. }\end{array}$ & - Limited mandate. \\
\hline
\end{tabular}


Table 1 Thirty-three actor types with possibilities to contribute to the reduction of antibiotic emissions from manufacturing. For each actor type, examples of their interests, possible actions and incentives and disincentives for those actions are listed (more details to be found in the main text and the supplementary material). Broadly common interests among actors, e.g. individual's/ employees desire to contribute to positive societal change (reducing pollution, improving public health) are implicit but have not been listed for each actor. For actors \#15,16,17, 21 and 22 we have listed Swedish examples of actors types (Continued)

\begin{tabular}{|c|c|c|c|c|}
\hline Actors & Interests & Actions & Incentives & Disincentives \\
\hline \#18 Public health agencies & $\begin{array}{l}\text { - Follow statutes } \\
\text { and directives as } \\
\text { defined by \#14; } \\
\text { - Good, affordable } \\
\text { health care. }\end{array}$ & $\begin{array}{l}\text { - Issue national treatment } \\
\text { guidelines (in cooperation } \\
\text { with \# 17) }\end{array}$ & $\begin{array}{l}\text { - Steering by national } \\
\text { government. }\end{array}$ & - Limited mandate. \\
\hline $\begin{array}{l}\text { \#19 Agencies committed to } \\
\text { public procurement: } \\
\text { Upphandlingsmyndigheten }\end{array}$ & $\begin{array}{l}\text { - Follow statutes } \\
\text { and directives as } \\
\text { defined by \#14; } \\
\text { - Good, affordable } \\
\text { health care. }\end{array}$ & $\begin{array}{l}\text { - Supporting \#20, } 21 \text { and } 22 \\
\text { to put pressure on \# } 1 \text { and } 2\end{array}$ & $\begin{array}{l}\text { - Steering by national } \\
\text { government. }\end{array}$ & - Limited power. \\
\hline \#20 Public hospitals and clinics & $\begin{array}{l}\text { - Follow statutes } \\
\text { and directives as } \\
\text { defined by } \# 14 \\
\text { and } \# 21 ; \\
\text { - Represent } \\
\text { interests of \#26 } \\
\& \# 28 ; \\
\text { - Effective } \\
\text { resource } \\
\text { allocation. }\end{array}$ & $\begin{array}{l}\text { - Apply environmental criteria } \\
\text { in procurement; } \\
\text { - Improve awareness. }\end{array}$ & - Regulation. & $\begin{array}{l}\text { - Pressure on cost-efficiency; } \\
\text { - Limited negotiating power. }\end{array}$ \\
\hline $\begin{array}{l}\text { \#21 Regional government } \\
\text { (county council) and their } \\
\text { regional medical products } \\
\text { committees (Läkemedels- } \\
\text { kommittér) }\end{array}$ & $\begin{array}{l}\text { - Represent } \\
\text { population; } \\
\text { - Protect public } \\
\text { health; } \\
\text { - Good, affordable } \\
\text { health care; } \\
\text { - Protect } \\
\text { economic } \\
\text { interests. }\end{array}$ & $\begin{array}{l}\text { - Steer \#20; } \\
\text { - Weigh in environmental } \\
\text { concerns in regional } \\
\text { treatment recommendations. }\end{array}$ & $\begin{array}{l}\text { - Political pressure by } \\
\text { citizens and interest } \\
\text { groups; } \\
\text { - National policies; } \\
\text { - Public health concerns. }\end{array}$ & - Limited power. \\
\hline $\begin{array}{l}\text { \#22 Central priority setting } \\
\text { organisation for drug } \\
\text { procurement (NT-rådet \& } \\
\text { Sveriges kommuner och } \\
\text { landsting, SKL) }\end{array}$ & $\begin{array}{l}\text { - Effective } \\
\text { resource } \\
\text { allocation; } \\
\text { - Good, affordable } \\
\text { health care. }\end{array}$ & $\begin{array}{l}\text { - Help counties act jointly and } \\
\text { effectively. }\end{array}$ & $\begin{array}{l}\text { - Steering by national } \\
\text { government. }\end{array}$ & - Limited mandate. \\
\hline $\begin{array}{l}\text { \#23 Privately funded and } \\
\text { operated clinics and hospitals }\end{array}$ & $\begin{array}{l}\text { - Profit; } \\
\text { - Promote and } \\
\text { protect health of } \\
\text { their patients. }\end{array}$ & $\begin{array}{l}\text {-Apply environmental criteria } \\
\text { when buying antibiotics. }\end{array}$ & $\begin{array}{l}\text { - Demands made by } \\
\text { subcontracting county } \\
\text { councils; } \\
\text { - Pressure from \#28, } 29 \text {. }\end{array}$ & - Very little negotiating power. \\
\hline \#24 Pharmacies & $\begin{array}{l}\text { - Profit; } \\
\text { - Reputation } \\
\text { concerns. }\end{array}$ & $\begin{array}{l}\text { - Take environmental } \\
\text { concerns into account when } \\
\text { purchasing antibiotics } \\
\text { (applicable to some } \\
\text { countries, not all); } \\
\text { - Improve awareness. }\end{array}$ & $\begin{array}{l}\text { - Media attention; } \\
\text { - Attracting costumers. }\end{array}$ & $\begin{array}{l}\text {-Little or no influence over what } \\
\text { antibiotics to provide through } \\
\text { governmental restrictions (in } \\
\text { some countries, but not all). }\end{array}$ \\
\hline \#25 Insurance companies & $\begin{array}{l}\text { - Profit; } \\
\text { - Reputation } \\
\text { concerns. }\end{array}$ & - Negotiate, pressure \# 1,2. & $\begin{array}{l}\text { - Financial considerations (e. } \\
\text { g. premiums, or taxes). }\end{array}$ & - Interest in lower prices. \\
\hline $\begin{array}{l}\text { \#26 Physicians and other } \\
\text { health care professionals }\end{array}$ & $\begin{array}{l}\text { - Economic } \\
\text { interests (in } \\
\text { some settings); } \\
\text { - Professional } \\
\text { ethos. }\end{array}$ & $\begin{array}{l}\text { - Pressure, primarily through } \\
\text { \#27. }\end{array}$ & $\begin{array}{l}\text { - Increased awareness. } \\
\text {-Pressure from lobby } \\
\text { groups, particularly \#1,2 and } \\
4 \text { (in some settings) }\end{array}$ & $\begin{array}{l}\text { Lack of information/ awareness } \\
\text { - Lack of interest/time; } \\
\text { - Lack of effective political } \\
\text { power. }\end{array}$ \\
\hline $\begin{array}{l}\text { \#27 Physician and other health } \\
\text { care professional organisations }\end{array}$ & $\begin{array}{l}\text { Represent } \\
\text { interests of \#26. }\end{array}$ & $\begin{array}{l}\text { - Pressure relevant policy } \\
\text { makers and institutions. } \\
\text { - Create awareness among } \\
\text { members, the public, } \\
\text { politicians and policy makers. }\end{array}$ & - Pressure by members. & $\begin{array}{l}\text { - Lack of effective political } \\
\text { power. }\end{array}$ \\
\hline
\end{tabular}


Table 1 Thirty-three actor types with possibilities to contribute to the reduction of antibiotic emissions from manufacturing. For each actor type, examples of their interests, possible actions and incentives and disincentives for those actions are listed (more details to be found in the main text and the supplementary material). Broadly common interests among actors, e.g. individual's/ employees desire to contribute to positive societal change (reducing pollution, improving public health) are implicit but have not been listed for each actor. For actors \#15,16,17, 21 and 22 we have listed Swedish examples of actors types (Continued)

\begin{tabular}{|c|c|c|c|c|}
\hline Actors & Interests & Actions & Incentives & Disincentives \\
\hline consumer country states & $\begin{array}{l}\text { medicines low; } \\
\text { - Access to } \\
\text { antibiotics. }\end{array}$ & $\begin{array}{l}\text { - Vote, exert political pressure; } \\
\text { - (When possible) buying } \\
\text { "environmentally certified" } \\
\text { antibiotics. }\end{array}$ & & $\begin{array}{l}\text { - Lack of interest; } \\
\text {-Increased costs for medicines; } \\
\text { - Lack of effective political } \\
\text { power. }\end{array}$ \\
\hline \#29 Patient organisations & $\begin{array}{l}\text { Represent } \\
\text { interests of \#28. }\end{array}$ & $\begin{array}{l}\text { - Pressure on county } \\
\text { governments or inter- } \\
\text { regional coordinating } \\
\text { bodies; } \\
\text { - Improve awareness. }\end{array}$ & - Pressure by members; & $\begin{array}{l}\text { - Lack of effective political } \\
\text { power. }\end{array}$ \\
\hline $\begin{array}{l}\text { \#30 Multinational governing } \\
\text { bodies (e.g. the EU) }\end{array}$ & $\begin{array}{l}\text { - Represent } \\
\text { member states; } \\
\text { - Streamlining the } \\
\text { national policies. }\end{array}$ & $\begin{array}{l}\text { - Regulate; } \\
\text { - Negotiate, pressure; } \\
\text {-Subsidize sustainable } \\
\text { practices; } \\
\text {-Research funding. }\end{array}$ & $\begin{array}{l}\text { - Political pressure; } \\
\text { - Treaties, multilateral } \\
\text { agreements, foreign } \\
\text { pressure. }\end{array}$ & $\begin{array}{l}\text { - Non-aligning interests between } \\
\text { member states; } \\
\text { - Lobbying; } \\
\text { - Lack of jurisdiction. Outside of } \\
\text { e.g. EU. }\end{array}$ \\
\hline $\begin{array}{l}\text { \#31 Agencies of multistate } \\
\text { bodies (such as the European } \\
\text { Medicines Agency) }\end{array}$ & $\begin{array}{l}\text { - Follow statutes } \\
\text { and directives as } \\
\text { defined by \#14. }\end{array}$ & $\begin{array}{l}\text { - Amend licensing } \\
\text { requirements (ERA) to } \\
\text { include risks for AMR } \\
\text { selection and production } \\
\text { emissions; } \\
\text { - Include environmental } \\
\text { considerations in GMP; } \\
\text { - facilitate transparency of } \\
\text { production chains. }\end{array}$ & - Steering by \#30. & $\begin{array}{l}\text { - Lack of research data to define } \\
\text { demands; } \\
\text { - Lack of jurisdiction outside of } \\
\text { e.g. EU. }\end{array}$ \\
\hline \#32 Media & $\begin{array}{l}\text { - Profit; } \\
\text { - Credibility; } \\
\text { - Public interest. }\end{array}$ & $\begin{array}{l}\text { - Improve awareness; } \\
\text { - Expose polluters; } \\
\text { - Demand action from the } \\
\text { majority of actors. }\end{array}$ & $\begin{array}{l}\text { - More viewers/readers; } \\
\text { - Curiosity; } \\
\text { - Increased credibility. }\end{array}$ & $\begin{array}{l}\text { - Opacity of productions chains; } \\
\text {-Lack of emission data. }\end{array}$ \\
\hline $\begin{array}{l}\text { \# } 33 \text { Scientific researchers and } \\
\text { universities }\end{array}$ & $\begin{array}{l}\text { - Reputation; } \\
\text { - Receive funding. }\end{array}$ & $\begin{array}{l}\text { - Generate knowledge; } \\
\text { - Educate and create } \\
\text { awareness among other } \\
\text { actors; } \\
\text { - Propose scientifically funded } \\
\text { actions for e.g. regulation } \\
\text { and procurement. }\end{array}$ & $\begin{array}{l}\text { - Curiosity } \\
\text { - Reputation; } \\
\text { - Funding; }\end{array}$ & $\begin{array}{l}\text { - Institutional barriers to } \\
\text { multidisciplinary and/or } \\
\text { international cooperation; } \\
\text { - Limited access to data and } \\
\text { samples from industry. }\end{array}$ \\
\hline
\end{tabular}

